Instituto Internacional de Investigación y Desarrollo Tecnológico Educativo INDTEC, C.A.

DOI: https://doi.org/10.29394/Scientific.issn.2542-2987.2020.5.16.9.179-193

OAI-PMH: http://www.indteca.com/ojs/index.php/Revista Scientific/oai

Artículo Original / Original Article

\title{
La evaluación formativa, una práctica eficaz en el desempeño docente
}

Autora: María Zoila Joya Rodríguez Universidad César Vallejo, UCV zoilajoyar2012@gmail.com Lima, Perú

\section{Resumen}

La evaluación formativa como proceso pedagógico cumple una función esencial para optimizar el proceso enseñanza-aprendizaje de los estudiantes, no obstante, es una práctica poco estudiada y aplicada en el quehacer educativo. Por tal razón, el objetivo de este estudio fue valorar el conocimiento, la didáctica e instrumentos de evaluación formativa en la práctica pedagógica de los docentes de secundaria de la Institución Educativa Sor Querubina de San Pedro de Surquillo, Lima, Perú. Mediante un diseño de InvestigaciónAcción de enfoque cualitativo, se recopilaron los datos informativos a través de entrevistas, grupo focal y observaciones de sesiones de clases. Se utilizó el software ATLAS.ti 7, para la revisión de los resultados obtenidos y en la triangulación se determinaron las categorías: mejora de los aprendizajes e instrumentos de evaluación. Como conclusión, se determinó que los docentes valoran y promueven, en sus sesiones de enseñanza, la evaluación formativa porque mejora las competencias de los alumnos con una evaluación permanente y una mayor participación en clase. Además, utilizan, en su mayoría, instrumentos de evaluación formativa como intervenciones orales, prácticas calificadas, trabajos colaborativos y rúbricas para evaluar exposiciones grupales de los estudiantes.

Palabras clave: evaluación formativa; docente de secundaria; práctica pedagógica.

Cómo citar este artículo:

Joya, M. (2020). La evaluación formativa, una práctica eficaz en el desempeño docente. Revista

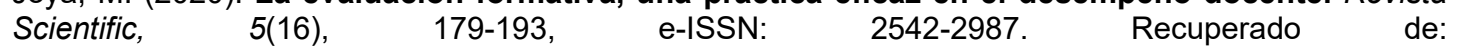
https://doi.org/10.29394/Scientific.issn.2542-2987.2020.5.16.9.179-193

Fecha de Recepción:

01-07-2019
Fecha de Aceptación:

21-01-2020
Fecha de Publicación:

05-05-2020 


\title{
Formative evaluation, an effective practice in teaching performance
}

\begin{abstract}
Formative evaluation as a pedagogical process fulfills an essential function to optimize the teaching-learning process of students, however, it is a practice little studied and applied in the educational task. For this reason, the objective of this study was to assess the knowledge, didactics and formative evaluation instruments in the pedagogical practice of secondary school teachers of the Sor Querubina Educational Institution of San Pedro de Surquillo, Lima, Peru. Through a Research-Action design of a qualitative approach, informational data was collected through interviews, focus group and class session observations. The ATLAS.ti 7 software was used, for the review of the results obtained and in the triangulation the categories were determined: improvement of learning and evaluation instruments. In conclusion, it was determined that teacher's value and promote, in their teaching sessions, formative evaluation because it improves the competencies of students with a permanent evaluation and greater participation in class. In addition, they mostly use formative assessment instruments such as oral interventions, qualified practices, collaborative and rubric work to evaluate group exposures of students.
\end{abstract} practice.

Keywords: formative evaluation; secondary school teachers; teaching

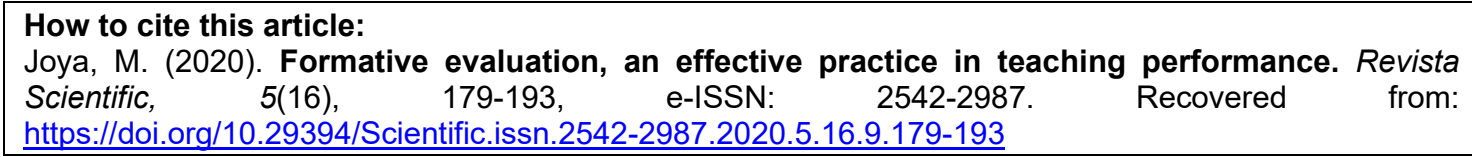

Date Received:

01-07-2019
Date Acceptance:

21-01-2020
Date Publication:

05-05-2020 


\section{Introducción}

Son pocas las investigaciones que se han realizado sobre la competencia de profesores en el empleo de instrumentos y estrategias de la evaluación formativa para medir el desarrollo de capacidades en los estudiantes. De acuerdo con Moreno, Candela y Bañuelos (2019): la práctica de evaluación formativa en el ámbito docente es un tema poco estudiado y existe escasa información sobre el conocimiento de este proceso en la formación de los docentes y otros profesionales.

En este sentido, Talanquer (2015): a través de las investigaciones educativas de las últimas décadas se ha comprobado que los métodos de enseñanza tradicionales que se basan en la transmisión de conocimientos a los estudiantes, no han sido efectivos para el desarrollo del proceso de sus aprendizajes. Asimismo, revelan que es muy importante el concurso de los estudiantes en la construcción de sus saberes y logro de competencias y capacidades. Para tal efecto, se deben implementar en el aula procesos pedagógicos y cognitivos en los que los alumnos sean el centro y protagonistas de los aprendizajes.

En este marco, la evaluación como proceso pedagógico ha variado su finalidad. De estar asociada a una cuantificación del aprendizaje con resultados numéricos constituye un proceso educativo que expresa a la vez cantidad y excelencia en el logro de los propósitos educativos. Parafraseando a Pérez, Enrique, Carbó y Gonzáles (2017a): esto brinda los suficientes datos para retroalimentar el proceso y realizar actividades de diagnóstico, estimulación y orientación a los estudiantes, a fin de que superen las dificultades en el aprendizaje.

Para Martínez-Mínguez, Moya, Nieva y Cañabate (2019): la evaluación como estrategia pedagógica, motiva a los educandos en el logro de sus saberes y los orienta hacia la mejora continua, informándoles sobre sus progresos en la consecución de sus competencias; en la práctica se convierte 
en la mejor actividad de aprendizaje. Exponen, Pérez, Enrique, Carbó y Gonzáles (2017b): es muy importante que, para optimizar los aprendizajes, los docentes deben tener una sólida formación pedagógica, estar actualizados y manejar con eficiencia las estrategias de evaluación formativa.

La evaluación tiene primordial influencia como proceso pedagógico que orienta la construcción del aprendizaje de los alumnos. Sin lugar a dudas condiciona la enseñanza y aprendizaje. Mencionando a García et al. (2013): evaluar es una acción ciertamente difícil en el crecimiento de la persona. Es un proceso cabal y metódico que permite recabar información rigurosa; es decir, la evaluación busca datos para tomar decisiones, exige uso de destrezas y dispositivos para reunir información.

Según Hortigúela, Pérez-Pueyo y Gonzáles-Calvo (2019a): hasta nuestros días subsiste en la práctica docente el considerar la evaluación como sinónimo de calificación. Esta confusión conlleva a realizar en el aula actividades evaluativas que no son nada pedagógicas. No todo lo evaluable tiene que ser calificable, entendiendo por calificación a una nota numérica al culminar un proceso; los nuevos enfoques educativos consideran que, la evaluación está asociada al aprendizaje. Señalando a Pasek y Mejía (2017): este tipo de evaluación es formativa y plantea una concepción constructivista del aprendizaje, en la que el estudiante estructura sus conocimientos a través de las actividades educativas que realiza; concebida así, la evaluación formativa es el proceso a través del cual el docente identifica los errores de los estudiantes, entiende sus causas y toma decisiones para superarlas, mejora tanto el aprendizaje de los estudiantes como su desempeño docente.

La Universidad Católica Sedes Sapientiae (UCSS, 2015): con la finalidad de contar con la información más precisa de evaluación formativa, se utilizan instrumentos y técnicas específicas. En la investigación se han considerado recursos poco formales como observaciones, diálogos e interrogaciones. Asimismo, técnicas más o menos formales en las que por ser 
discretas el estudiante no piensa que le están evaluando. Para Segura (2018): se consideran entre ellas, la relación de anécdotas, reportes de clases y las rúbricas entre otros. Además, Díaz y Barriga (2002): indican que también se emplean técnicas formales como los exámenes escritos, las tareas y los mapas conceptuales.

Para el efecto, se desarrollaron jornadas de capacitación pedagógica, actualizando a los docentes en los principios y cualidades de la evaluación formativa, así como el análisis reflexivo acerca de sus desempeños para reconocer sus fortalezas y debilidades en la tarea evaluativa, y en sus determinaciones para cambiar sus prácticas pedagógicas. Se debe entender, según Hortigúela, Pérez-Pueyo y Gonzáles-Calvo (2019b): que la evaluación formativa es el medio más eficaz para la mejora de los aprendizajes y para optimizar la práctica docente.

Teniendo en consideración lo anteriormente planteado, se pretende con la siguiente investigación descubrir qué tanto conocen los docentes de secundaria de la I.E. Sor Querubina de San Pedro, de la evaluación formativa en la adquisición del conocimiento y el manejo de métodos, instrumentos y técnicas de este proceso. En consecuencia, los objetivos del presente estudio son: a). Valorar el conocimiento y práctica de la evaluación formativa en los docentes de secundaria de la I.E. Sor Querubina de San Pedro; y b). Reconocer la didáctica y los medios de evaluación formativa que utilizan los profesores en sus sesiones de aprendizaje.

\section{Metodología}

Mencionando a Hernández, Fernández y Baptista (2010): se realizó una investigación cualitativa, orientados al conocimiento de la persona en sus vivencias cotidianas, en el contexto social y sus relaciones interpersonales; se detectó el problema, se hizo un planteamiento y se recolectó datos para elaborar un plan con objetivos, estrategias y acciones programadas en el 
tiempo, se puso en marcha el plan donde hubo ajustes, decisiones, volver a definir y dar nuevos diagnósticos.

El presente estudio, de acuerdo con Latorre (2005): se basa en la metodología Investigación-Acción que es una estrategia metodológica a través de la cual el docente reflexiona sobre su desempeño pedagógico, generando una autocrítica y la determinación de retroalimentar su acción didáctica para optimizar el aprendizaje de sus alumnos. La Investigación-Acción, como lo exponen Boggino y Rosekrans (2004): utiliza diversidad de métodos cualitativos, cuantitativos, técnicas e instrumentos para el logro de la información, observación y evaluación deseadas.

El diseño que se aplicó fue Investigación - Acción: se hizo un análisis crítico de la práctica educativa de los docentes de secundaria de la I.E. Sor Querubina de San Pedro, en el uso de la evaluación formativa en sus clases. Para Requena (2018): los docentes son los actores, los participantes que aprendieron a identificar las causas de sus problemas y encontraron posibles soluciones para mejorar su desempeño docente.

Se recogieron testimonios y manifiestos de los docentes y estudiantes en las entrevistas, del Grupo Focal "focus group" y observaciones de sesiones de clases. Se entrevistaron estudiantes de secundaria del Colegio Sor Querubina de San Pedro del distrito de Surquillo y se realizó un grupo focal con otros estudiantes de secundaria de $3^{\circ}$ y $4^{\circ}$ grado y se observaron sesiones de clases de los docentes de Matemática y Ciencia y Tecnología. Los testimonios y observaciones fueron transcritos en un cuaderno de campo. Con toda la información recogida se formó redes hermenéuticas con el software ATLAS. Ti 7.

Para la identificación de las categorías, se codificaron las fuentes que fueron el Grupo Focal con seis estudiantes, las entrevistas con seis docentes y las fichas de observaciones de clases de los docentes. En este sentido, y como presenta Galeano (2004): se hizo la triangulación del análisis de las 
conclusiones de cada fuente, se formó el constructo que se dedujo de la triangulación de los códigos encontrados en las fuentes. Se trabajó con seis (6) estudiantes y seis (6) docentes, después de la construcción del constructo y categorización de la información con la ayuda de la codificación de las fuentes y de los mapas semánticos elaborados con el programa ATLAS. Ti 7, se pudo determinar las Categorías: instrumentos de evaluación y mejora de los aprendizajes.

\section{Análisis e interpretación de los resultados}

En la tabla 1, podemos visualizar la categorización de la información obtenida en las observaciones de clases de los docentes de Matemática y Ciencias donde se pudo verificar que en la categoría mejora de los aprendizajes, los estudiantes fueron motivados por los docentes, participando activamente con entusiasmo y espontaneidad. Los estudiantes que encontraban sentido e interés en lo que aprendían, daban sus aportes, comentaban y preguntaban en las sesiones de clases.

Teniendo en cuenta que las sesiones han sido de Matemática y Ciencias, en el momento de la aplicación de lo aprendido, los estudiantes desarrollaron ejercicios y problemas planteados por el profesor para constatar si hubo aprendizaje. Los docentes observados, cumplían casi en su totalidad con monitorear y hacer un seguimiento al trabajo de los estudiantes, retroalimentando sus aprendizajes.

Con respecto a la supervisión del trabajo grupal, cuatro docentes supervisaban el trabajo en grupo y realizaba el monitoreo correspondiente, esta estrategia en clase promovía la interacción en el aprendizaje, propiciaba el trabajo colaborativo y enriquecía los conocimientos de los estudiantes. 
Instituto Internacional de Investigación y Desarrollo Tecnológico Educativo INDTEC, C.A.

DOI: https://doi.org/10.29394/Scientific.issn.2542-2987.2020.5.16.9.179-193

OAI-PMH: http://www.indteca.com/ojs/index.php/Revista_Scientific/oai

Artículo Original / Original Article

Tabla 1. Categorización de la información.

\begin{tabular}{|c|c|c|c|}
\hline Categorías & Sub categorías & $\begin{array}{l}\text { Constructo desde la } \\
\text { Observación de clases }\end{array}$ & $\begin{array}{c}\mathrm{N}^{\circ} \\
\text { Docentes }\end{array}$ \\
\hline \multirow{5}{*}{$\begin{array}{l}\text { MEJORA DE LOS APRENDIZAJES: } \\
\text { El aprendizaje es un proceso de } \\
\text { adquisición de conocimientos, } \\
\text { habilidades, valores y actitudes, } \\
\text { posibilitado mediante el estudio, la } \\
\text { enseñanza o la experiencia. }\end{array}$} & $\begin{array}{l}\text { Procesos de } \\
\text { aprendizaje }\end{array}$ & $\begin{array}{l}\text { El docente desarrolla los } \\
\text { procesos para que el } \\
\text { estudiante aprenda. }\end{array}$ & 2 \\
\hline & $\begin{array}{l}\text { Motiva a los } \\
\text { estudiantes }\end{array}$ & $\begin{array}{c}\text { El profesor incentivo a los } \\
\text { estudiantes para que } \\
\text { aprendan. }\end{array}$ & 3 \\
\hline & $\begin{array}{l}\text { Participación } \\
\text { activa de los } \\
\text { estudiantes }\end{array}$ & $\begin{array}{c}\text { Los estudiantes cuando } \\
\text { están motivados participan } \\
\text { activamente de la sesión } \\
\text { de clase. }\end{array}$ & 4 \\
\hline & $\begin{array}{c}\text { Orienta el } \\
\text { trabajo } \\
\text { individual }\end{array}$ & $\begin{array}{l}\text { Los docentes monitorean el } \\
\text { trabajo del estudiante. }\end{array}$ & 4 \\
\hline & $\begin{array}{l}\text { Supervisa el } \\
\text { trabajo grupal }\end{array}$ & $\begin{array}{c}\text { El docente monitorea el } \\
\text { trabajo en grupo de los } \\
\text { estudiantes. }\end{array}$ & 4 \\
\hline \multirow{6}{*}{$\begin{array}{l}\text { INSTRUMENTOS DE EVALUACIÓN: } \\
\text { LoS instrumentos de evaluación se } \\
\text { constituyen en el soporte físico que se } \\
\text { emplea para recoger la información } \\
\text { sobre los aprendizajes esperados del } \\
\text { estudiante. }\end{array}$} & $\begin{array}{l}\text { Intervenciones } \\
\text { orales }\end{array}$ & $\begin{array}{l}\text { Los estudiantes participan } \\
\text { activamente en clase } \\
\text { haciendo intervenciones } \\
\text { orales a las preguntas } \\
\text { formuladas por el docente. }\end{array}$ & 6 \\
\hline & $\begin{array}{l}\text { Prácticas } \\
\text { Calificadas }\end{array}$ & $\begin{array}{l}\text { Los estudiantes desarrollan } \\
\text { prácticas escritas y estas } \\
\text { son calificadas por el } \\
\text { docente para evaluar el } \\
\text { proceso del aprendizaje. }\end{array}$ & 3 \\
\hline & $\begin{array}{l}\text { Trabajos } \\
\text { Grupales }\end{array}$ & $\begin{array}{l}\text { Los estudiantes realizan un } \\
\text { trabajo con otros } \\
\text { compañeros para apoyarse } \\
\text { y consolidar aprendizajes. }\end{array}$ & 4 \\
\hline & $\begin{array}{l}\text { Revisión de } \\
\text { cuadernos }\end{array}$ & $\begin{array}{c}\text { El cuaderno registra el } \\
\text { avance teórico de la sesión } \\
\text { de clase como evidencia } \\
\text { de lo avanzado. }\end{array}$ & 2 \\
\hline & $\begin{array}{l}\text { Uso de Rúbricas } \\
\text { para evaluar. }\end{array}$ & $\begin{array}{c}\text { Guías con bases a criterios } \\
\text { específicos sobre la } \\
\text { actividad a evaluar en una } \\
\text { exposición. }\end{array}$ & 4 \\
\hline & $\begin{array}{l}\text { Registra } \\
\text { evaluación }\end{array}$ & $\begin{array}{c}\text { El docente anota las } \\
\text { intervenciones de } \\
\text { evaluación de los } \\
\text { estudiantes. }\end{array}$ & 4 \\
\hline
\end{tabular}

Fuente: La Autora (2019).

De las observaciones de las sesiones de clases, en los docentes de Ciencias y Matemática, se pudo apreciar que elegir los instrumentos de evaluación, constituye un reto a la competencia y creatividad de los docentes. 
Como podemos observar en la tabla 1, estos instrumentos debieron ser variados en las diferentes sesiones, pertinentes a los temas, motivadores del aprendizaje.

Después del taller desarrollado y las coordinaciones de área, los docentes observados utilizaban los instrumentos para evaluar a los estudiantes quienes conocían de antemano los recursos con que serían medidos sus aprendizajes.

Las intervenciones orales, fueron el instrumento tradicional que más utilizaban los docentes observados, cuatro (4) docentes registraban sus intervenciones en su documento auxiliar de evaluación. Cuatro (4) docentes, utilizaron el trabajo grupal como estrategia para evaluar a los estudiantes, así los alumnos interactuaban, compartían saberes y consolidaban sus aprendizajes, usaban rúbricas para evaluar las exposiciones de los trabajos grupales las cuales elaboraron en grupos de docentes.

Se observó, que los docentes aplicaban prácticas calificadas como instrumentos de evaluación. Pese a que el cuaderno de trabajo de los estudiantes no es un instrumento para evaluar el aprendizaje, pero, sin embargo, el que "esté al día" o tenga todos los ejercicios resueltos, para los docentes es una evidencia del logro del aprendizaje. Aparecía en el registro del Profesor como un criterio de evaluación.

\section{Conclusiones}

Tomemos ahora como referencia, la investigación realizada por Rosales (2018): en la que concluye que los profesores con alto nivel de información sobre evaluación formativa, son muy competentes en su desempeño pedagógico, y atribuye el éxito de un docente en su práctica si está bien capacitado. Podemos compararlo con nuestra investigación en la que los docentes fueron capacitados por especialistas en la elaboración de instrumentos de evaluación formativa y los docentes se sintieron más seguros 
de aplicar lo aprendido en grupo.

Sin embargo, Portocarrero (2017): en su estudio muestra en sus conclusiones que los beneficios de la evaluación formativa influyen tanto en la enseñanza como en el aprendizaje, y los educadores tomaron en cuenta la metodología de la evaluación formativa de otros profesores y así enriquecieron su práctica docente. Reconozco que este es un trabajo que queda pendiente y que es muy gratificante para los que participan propiciando el monitoreo $u$ observación de sesiones de clases entre los propios docentes, ya que hace fortalecer los conocimientos de evaluación formativa entre los profesores.

Debemos tener en cuenta, que la investigación de Pérez, Enrique, Carbó y Gonzales (2017c): en su conclusión nos dice que un docente competente es capaz de emplear una adecuada evaluación formativa aplicando métodos diversos, dirigidos a alcanzar novedosas metas de aprendizaje. Coincide plenamente con nuestra investigación porque docentes capacitados y comprometidos promueven una práctica de evaluación formativa que mejora los aprendizajes.

Por último, es necesario mencionar el estudio de Álvarez y Zamora (2017): quien menciona en su conclusión general que promovidas e incorporadas la metodología de la evaluación formativa en los profesores se está contribuyendo a la calidad de la educación. Lo señalado por estos autores nos confirma lo que se concluyó en nuestra investigación que los docentes de secundaria de la I.E. Sor Querubina de San Pedro, reconocen, implementan y utilizan estrategias de una evaluación formativa en el desarrollo de sus sesiones de aprendizaje, valorando así, este proceso pedagógico y retroalimentando el aprendizaje en nuestros estudiantes peruanos, llegando a las siguientes conclusiones:

Primera: Los profesores del Nivel de secundaria del Colegio "Sor Querubina de San Pedro", valoran y promueven en su práctica de evaluación formativa porque mejora los aprendizajes con una evaluación permanente y 
una mayor participación de los estudiantes en clase.

Segunda: Los docentes de educación secundaria, utilizan, en su mayoría, instrumentos de evaluación formativa como intervenciones orales, prácticas calificadas, trabajos grupales y rúbricas para valorar exposiciones grupales de los estudiantes.

Al no existir material de investigación suficiente en relación con estrategias e instrumentos de evaluación formativa, se encontró dificultades para aplicar con entera eficacia la metodología de Investigación-Acción, toda vez que los docentes monitoreados no evidenciaban plena competencia en este tema.

Se consideró necesario, fomentar en las Instituciones Educativas, jornadas de capacitación en este sentido y promover entre los docentes, círculos de calidad que propongan estrategias y construyan instrumentos de evaluación formativa para diferentes áreas curriculares, particularmente para Ciencias y Matemática.

\section{Referencias}

Álvarez, A., \& Zamora, O. (2017). Propuesta de intervención dirigida a fomentar la promoción e integración de estrategias de evaluación formativa del aprendizaje en el profesorado del Instituto Fernando Salazar Martínez del municipio de Nagarote-Nicaragua. Tesis. Managua, Nicaragua: Universidad Centroamericana-UCA. Recuperado de: http://repositorio.uca.edu.ni/4685/

Boggino, N., \& Rosekrans, K. (2004). Investigación-Acción: reflexión crítica sobre la práctica educativa. Santa Fe, Argentina: Homo Sapiens Ediciones.

Díaz, F., \& Barriga, A. (2002). Estrategias docentes para un aprendizaje significativo: Una interpretación constructiva. Técnicas e Instrumentos de Evaluación. México: McGraw-Hill. 
Galeano, M. (2004). Diseño de Proyectos de Investigación Cualitativa. Primera edición, ISBN: 958-8173-78-7. Medellín, Colombia: Fondo Editorial Universidad EAFIT. Recuperado de:

https://es.slideshare.net/juancarlosgomezjaramillo9/diseo-deproyectos-en-la-inv-cualitativa-maria-eumelia-galeano

García, N., Nicolás, R., Gómez, I., Fuentes, M., Reséndiz, N., Sandoval, M..., \& Díaz, M. (2013). El Enfoque formativo de la evaluación. Primera edición, ISBN: 978-607-467-285-5. México: Secretaría de Educación Pública.

Hernández, R., Fernández, C., \& Baptista, P. (2010). Metodología de la Investigación. 5ta edición. México, D.F.: Interamericana Editores, S.A. Hortigúela, D., Pérez-Pueyo, Á., \& Gonzáles-Calvo, G. (2019a,b). Pero... ¿A qué nos Referimos Realmente con la Evaluación Formativa y Compartida?: Confusiones Habituales y Reflexiones Prácticas. Revista Iberoamericana de Evaluación Educativa, 12(1), 13-27, e-ISSN: 1989-0397. Recuperado de:

http://dx.doi.org/10.15366/riee2019.12.1.001

Latorre, A. (2005). Investigación-Acción: Conocer y cambiar la práctica educativa. 3ra. Edición, ISBN 10: 84-7827-292-1; ISBN 13: 978-847827-292-1. Barcelona, España: Editorial Graó.

Martínez-Mínguez, L., Moya, L., Nieva, C., \& Cañabate, D. (2019). Percepciones de Estudiantes y Docentes: Evaluación Formativa en Proyectos de Aprendizaje Tutorados. Revista Iberoamericana de Evaluación Educativa, 12(1), 59-84, e-ISSN: 1989-0397. Recuperado de: http://dx.doi.org/10.15366/riee2019.12.1.004

Moreno, J., Candela, A., \& Bañuelos, P. (2019). Evaluaciones Formativas en el Aula: Análisis Discursivo de la Actividad de Retroalimentación en la Práctica Supervisada de Psicólogos Educativos en Formación. Revista Iberoamericana de Evaluación Educativa, 12(1), 
121-137, e-ISSN: 1989-0397. Recuperado de:

https://doi.org/10.15366/riee2019.12.1.007

Pasek, E., \& Mejía, M. (2017). Proceso General para la Evaluación

Formativa del Aprendizaje. Revista Iberoamericana de Evaluación

Educativa, 10(1), 177-193, e-ISSN: 1989-0397. Recuperado de:

http://dx.doi.org/10.15366/riee2017.10.1.009

Portocarrero, F. (2017). Implementación de estrategias de evaluación formativa en el nivel primario del Colegio Peruano Norteamericano

Abraham Lincoln. Tesis. Lima, Perú: Facultad de Ciencias de la

Educación de la Universidad de Piura. Recuperado de:

https://hdl.handle.net/11042/2886

Pérez, M., Enrique, J., Carbó, J., \& Gonzáles, M. (2017a,b,c). La evaluación formativa en el proceso enseñanza aprendizaje. EDUMECENTRO, 9(3), 263-283, e-ISSN: 2077-2874. Recuperado de:

http://www.revedumecentro.sld.cu/index.php/edumc/article/view/989

Requena, Y. (2018). Investigación Acción Participativa y Educación

Ambiental. Revista Scientific, 3(7), 289-308, e-ISSN: 2542-2987. Recuperado de:

https://doi.org/10.29394/Scientific.issn.2542-2987.2018.3.7.15.289-308

Rosales, M. (2018). El nivel de conocimiento sobre evaluación formativa en la práctica de la labor docente de una Institución Educativa Secundaria de Trujillo, 2017. Tesis. Perú: Escuela de Posgrado de la Universidad César Vallejo. Recuperado de:

http://repositorio.ucv.edu.pe/handle/UCV/11767

Segura, M. (2018). La función formativa de la evaluación en el trabajo escolar cotidiano. Revista Educación, 42(1), 1-19, ISSN: 0379-7082; e-ISSN: 2215-2644. Recuperado de:

http://dx.doi.org/https://dx.doi.org/10.15517/revedu.v42i1.22743

Talanquer, V. (2015). La importancia de la evaluación formativa. Educación 
OAI-PMH: http://www.indteca.com/ojs/index.php/Revista_Scientific/oai

\section{Artículo Original / Original Article}

Química, 26(3), 177-179, ISSN: 0187-893X; e-ISSN: 1870-8404.

Recuperado de: http://dx.doi.org/10.1016/j.eq.2015.05.001

UCSS (2015). Guía para la elaboración del proyecto de tesis. Lima, Perú:

Facultad de Ciencias de la Salud de la Universidad Católica Sedes

Sapientiae. Recuperado de: http://www.ucss.edu.pe/images/fcs/guia- 


\section{Artículo Original / Original Article}

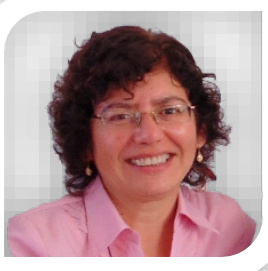

\section{María Zoila Joya Rodríguez}

e-mail: zoilajoyar2012@gmail.com

Nacida en Lima, Perú, el 2 de febrero del año 1963.

Doctora en Educación; Magister en Administración de la Educación, en la Universidad César Vallejo (UCV); Licenciada en Educación Secundaria en la especialidad de Matemática y Física, en el Instituto Pedagógico Nacional de Monterrico (IPNM). Actualmente me desempeño como Coordinadora Académica en la Institución Educativa "Sor Querubina de San Pedro".

El contenido de este manuscrito se difunde bajo una Licencia de Creative Commons ReconocimientoNoComercial-Compartirlgual 4.0 Internacional 